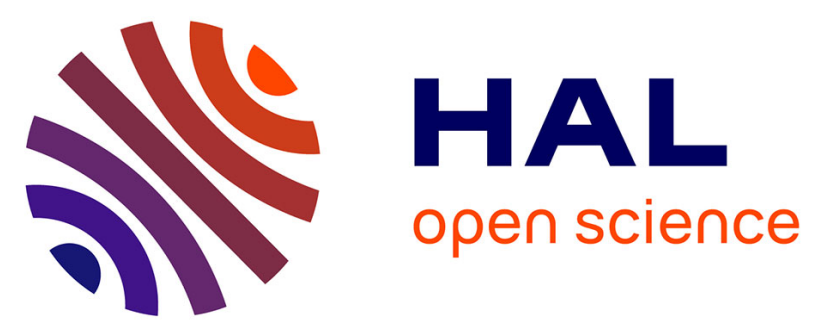

\title{
Systematic MRI in NF1 children under six years of age for the diagnosis of optic pathway gliomas. Study and outcome of a French cohort
}

Gaëlle Blanchard, Marie-Pierre Lafforgue, François Lion-François, Isabelle Kemlin, Diana Rodriguez, Pierre Castelnau, Maryline Carneiro, Pierre Meyer, Francois Rivier, Sébastien Barbarot, et al.

\section{To cite this version:}

Gaëlle Blanchard, Marie-Pierre Lafforgue, François Lion-François, Isabelle Kemlin, Diana Rodriguez, et al.. Systematic MRI in NF1 children under six years of age for the diagnosis of optic pathway gliomas. Study and outcome of a French cohort. European Journal of Paediatric Neurology, 2016, 20 (2), pp.275 - 281. 10.1016/j.ejpn.2015.12.002 . hal-01803195

\section{HAL Id: hal-01803195 \\ https://hal.umontpellier.fr/hal-01803195}

Submitted on 29 Nov 2019

HAL is a multi-disciplinary open access archive for the deposit and dissemination of scientific research documents, whether they are published or not. The documents may come from teaching and research institutions in France or abroad, or from public or private research centers.
L'archive ouverte pluridisciplinaire HAL, est destinée au dépôt et à la diffusion de documents scientifiques de niveau recherche, publiés ou non, émanant des établissements d'enseignement et de recherche français ou étrangers, des laboratoires publics ou privés. 


\section{Systematic MRI in NF1 children under six years of age for the diagnosis of optic pathway gliomas. Study and outcome of a French cohort}

\section{Gaelle Blanchard ${ }^{a}$, Marie-Pierre Lafforgue ${ }^{b}$, Laurence Lion-François ${ }^{a}$,}

Isabelle Kemlin c, Diana Rodriguez ${ }^{\mathrm{c}, d, e}$, Pierre 1 Maryline Carneiro ${ }^{i}$, Pierre Meyer ${ }^{i}$, François Riv Yves Chaix $b, m, n, *$, The NF France network

เu ${ }^{f, g, h}$, Sébastien Barbarot ${ }^{\text {, }}$

a Service de neurologie pédiatrique, Hôpital Femme, Mère, Enfant, Bron, France

${ }^{\mathrm{b}}$ Hopital des Enfants, CHU Purpan, Toulouse, France

c AP-HP, Service de Neurologie Pédiatrique et Centre de Référence des Neurofibromatoses, Hôpital Armand

Trousseau, CHU Paris Est, Paris, France

' Sorbonne Universités, UPMC Univ Paris 06, Paris, France

e Inserm U1141, Paris, France

${ }^{\mathrm{f}}$ Unité de Neuropédiatrie et Handicaps, Hôpital d'Enfants Gatien de Clocheville, CHU de Tours, France

g Inserm, Imagerie et Cerveau, UMR 930, CHRU de Tours, Hôpital Bretonneau, Tours, France

${ }^{\mathrm{h}}$ Université de Tours, François Rabelais, Tours, France

${ }^{\mathrm{i}}$ CHRU de Montpellier, Neuropédiatrie \& Centre de Référence des Troubles du Langage, Montpellier, France

'Université de Montpellier, UFR de Médecine, Montpellier, France

${ }^{\mathrm{k}}$ U1046 Inserm, UMR9214 CNRS, PhyMedExp, Université de Montpellier, Montpellier, France

${ }^{1}$ Clinique Dermatologique, CHU Hôtel-Dieu, Nantes, France

${ }^{\mathrm{m}}$ Inserm, Imagerie Cérébrale et Handicaps Neurologiques UMR 825, CHU Purpan, Toulouse, France

${ }^{\mathrm{n}}$ Université de Toulouse III - Paul Sabatier, Toulouse, France

\section{A R T I C L E I N F O}

Article history:

Received 28 June 2015

Received in revised form

13 November 2015

Accepted 2 December 2015

Keywords:

Neurofibromatosis type 1 (NF1)

MRI

Optic gliomas

Children

\begin{abstract}
A B S T R A C T
Background/Purpose: Optic pathway glioma (OPG) is the most common central nervous system tumor in children with neurofibromatosis type 1 (NF1), affecting $15-20 \%$ of patients. We reviewed the medical records of children systematically screened by ophthalmologic and MRI examinations to determine the influence of screening on the therapeutic management of children with OPG.

Methods: Data were collected on 306 newly diagnosed cases screened with systematic MRI from January 2001 to July 2007. In the OPG group, we distinguished the asymptomatic or symptomatic groups according to their initial status.

Results: Forty-five patients had confirmed OPG (14.7\%). Thirty-six patients $(80 \%)$ were asymptomatic and nine $(20 \%)$ were symptomatic at the time of diagnosis with visual symptoms in six cases. The average age at OPG diagnosis was 3.4 years with six patients (13\%) over six years old. Average follow-up was 7.7 years. Progression was observed in 16
\end{abstract}

* Corresponding author. Inserm, Hôpital des Enfants, Centre Hospitalier Universitaire de Toulouse, CHU Purpan, Place du Dr Baylac, F-31059 Toulouse Cedex 9, France. Tel.: +33(0)5 34558576.

E-mail address: chaix.y@chu-toulouse.fr (Y. Chaix). 
cases (35\%). Most patient conditions were managed conservatively (87\%). Six children (13\%) were treated with chemotherapy due to worsening visual function. All of these children had severe or mild visual impairment at the end of follow-up.

Conclusion: Our study does not support a clear benefit of systematic MRI screening in NF1 children under six years old. Systematic neuroimaging in our study did not influence therapeutic management. Although OPG diagnosis was made early, treatment with chemotherapy did not improve the final visual outcome. If MRI remains the best tool for the diagnosis of cerebral and spinal pathologies in the NF1 population, our current study questions the usefulness of systematic MRI screening for OPG diagnosis. Conversely, this study suggests that the indication of neuroimaging should be dictated by the results of annual clinical and ophthalmological assessments.

\section{Introduction}

Neurofibromatosis type 1 (NF1) is a multisystemic, inherited, autosomal dominant disorder with an estimated prevalence of 1 in $3500 .{ }^{1}$ The gene of this neurocutaneous disorder, a tumor suppressor gene, was cloned on the long arm of chromosome 17 (17q11.2) in 1990. The diagnostic criteria for NF1 are clinical, established on a National Institutes of Health consensus in 1987,2 re-evaluated in 1997. NF1 prognosis remains unpredictable, with the possibility of complications affecting various organs. One of the most common tumors in patients with NF1 is optic pathway glioma (OPG). It occurs mainly in childhood under six years of age with an estimated frequency of $15 \%-20 \%$ in NF1 children revealed by systematic neuroimaging (MRI). 3,4 Despite the high frequency of the tumor in this population, routine MRI screening has not been recommended in asymptomatic NF1 children. ${ }^{5}$ This recommendation is based on a longitudinal study ${ }^{3}$ showing that the majority of OPGs detected are asymptomatic without radiological progression, the fact that MRI cannot differentiate progressive and non-progressive forms of OPG, and finally no clear evidence exists to show that earlier intervention changes the prognosis for these patients. ${ }^{5}$ Conversely, the NF1 Optic Pathway Glioma Task Force recommends that all newly diagnosed NF1 patients under six years of age have full yearly ophthalmological examinations with assessment of visual acuity, visual fields, and funduscopy and slit-lamp examinations. In 2001, members of a French medical network devoted to $\mathrm{NF}^{6}{ }^{6}$ elaborated recommendations for the management of the disease. The group considered that MRI for early detection of OPG in young children should remain controversial because of the difficulty of obtaining complete and reliable ophthalmologic examinations in these children, especially in children under six years of age or in children with cognitive impairment. Accordingly, the French NF1 expert group recommended annual systematic ophthalmologic assessment in all NF1 children and systematic MRI in all newly diagnosed NF1 children under six years of age and/or in children with cognitive impairment. Twelve years after these recommendations were made, this study aimed to review the medical records of the children systematically screened by ophthalmologic and MRI examinations and to determine the influence of this screening on the therapeutic management of children with OPG.

\section{Materials and methods}

This multicentric retrospective study was conducted between 2001 and 2007 in six French Pediatrics Multidisciplinary Neurofibromatosis Centers (Lyon, Montpellier, Nantes, Armand Trousseau Hospital in Paris, Toulouse and Tours). All these centers followed the guidelines recommended in 2001 and published in 2002 by the French NF1 expert group. ${ }^{6}$ All children referred to one of these six multidisciplinary centers with a new NF1 diagnosis since January 2001 (from January 2001 to July 2007) were included in the study. The inclusions were discontinued after 2007 for two reasons: the necessity for sufficient follow-up and because after this time, MRI screening was less systematic in some centers. NF1 diagnosis was based on clinical criteria defined by the NF1 Consensus Conference. ${ }^{2}$ All children under six years of age diagnosed with NF1 were screened systematically by brain and orbit MRI and ophthalmologic examination. Depending on the center, MRI was performed with or without sedation and repeated two years later if the results were normal. Ophthalmologic examinations included assessments of visual acuity, funduscopy, and slit-lamp examinations.

The data reviewed from medical records were age at NF1 diagnosis, age at OPG diagnosis, NF1 characteristics (sporadic or familial form, clinical manifestations, etc.), ophthalmologic screening results, and brain and orbit MRI results with location of the tumor. OPG is defined on MRI by an enlarged T1 isointense optic nerve with a T2 hyperintense signal with variable gadolinium enhancement. MRI provides assessment of the posterior extent of the tumor. We differentiated groups of children according to the MRI results as children with OPG and without OPG. For the group of children without OPG at the first examination, we recorded the annual examination and determined if they had been given a second MRI. In the OPG group, children were classified according to their status as either being asymptomatic or initially symptomatic. The asymptomatic OPG group represented children who had had a "baseline" MRI performed as a screening tool. In the symptomatic OPG group, patients had brain MRI to investigate a specific clinical sign (neurologic, ophthalmologic, or endocrinologic) that could be attributed to the presence of OPG. We reviewed the successive ophthalmologic and MRI examinations (studies at three-to six-month intervals, the first 12 
months, and then yearly). A decrease in visual acuity or the appearance of endocrinologic symptoms related to the tumor were considered as clinical OPG progression. Visual function impairment was defined as a visual loss of two degrees. We considered that visual deficit was severe when visual acuity was less than $2 / 10$ and mild when visual acuity was between $2 / 10$ and $8 / 10$ and/or when the visual field was altered. Increase of tumor volume on MRI studies was considered as radiological progression.

We compared asymptomatic and symptomatic groups: mean values or medians were used as continuous variables and frequencies were used as categorical variables. Nonparametric tests were used to compare continuous variables and the Pearson's chi-squared test for categorical variables; the level significance corresponded to a value of $\mathrm{p}<0.05$.

\section{Results}

\subsection{All groups}

The study was conducted from January 2001 to July 2007 and included 306 newly diagnosed children screened with systematic MRI in the five different centers. OPG was identified by MRI in 45 children (14.7\%), which included $36(80 \%)$ asymptomatic and nine $(20 \%)$ symptomatic children at the time of diagnosis. Two children with a normal first MRI developed symptomatic OPG subsequently. The general characteristics of the population are summarized in Table 1 . The average age at NF1 diagnosis was three years, the sex ratio was 1:1, and $42 \%$ had a family history of NF1. When the first MRI screening was normal, a second MRI was performed two years later in 45 children, which was normal in all cases. The first MRI was performed before the age of three years in $47 \%$ of case. The average age at OPG diagnosis was 3.4 years with six patients (13\%) diagnosed after the age of six years. Among these patients, only one girl was symptomatic. She was lost to followup. Of the 45 OPG patients, 20 patients (44\%) had isolated optic nerve involvement of one or both nerves, $16(35 \%)$ had both nerve and chiasma involvement, and six (13\%) had isolated chiasma enlargement. Only three patients $(7 \%)$ had postchiasmal extension. Four OPG patients had other CNS lesions. The MRI results were consistent with two cerebellar pilocytic astrocytomas and two non-evolutive pontine gliomas. Nine patients were symptomatic at the time of MRI screening: almost all the children with symptomatic OPG had isolated or associated ophthalmologic symptoms - strabismus was recorded in five cases, decreased visual acuity in
Table 2 - Comparison evolution for asymptomatic and symptomatic GVO Groups.

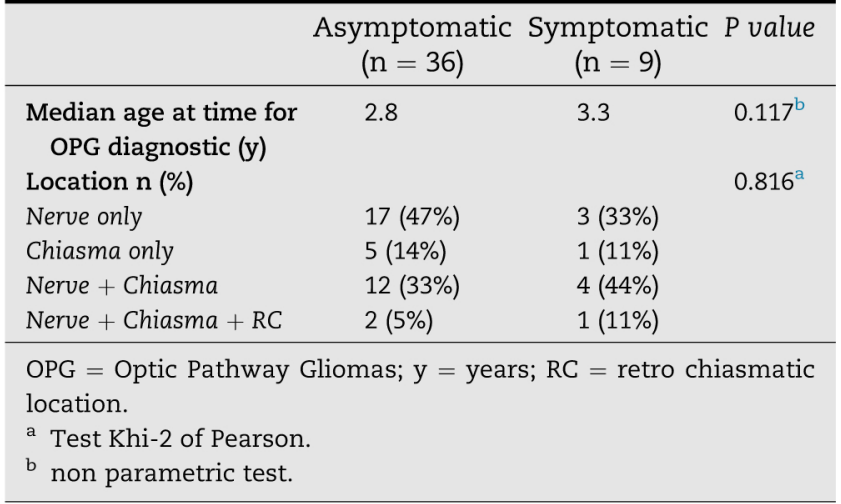

three cases, papillary abnormalities in one case, and proptosis in one case. Five (5/45) patients had evidence of optic disc atrophy or swelling on examination.

\subsection{Symptomatic versus asymptomatic OPG at diagnosis (Table 2)}

The symptomatic group had a higher proportion of patients with a positive family history; however, the difference was not statistically significant. Average age at diagnosis for asymptomatic children was 3.4 years (median $=2.8$ ) and 3.5 years (median $=3.3$ ) for symptomatic children. This difference in age of diagnosis between the two groups was not statistically significant. The asymptomatic group had a higher proportion of patients with isolated optic nerve involvement $(47 \%$ vs. $33 \%$ ), whereas the symptomatic group had a higher proportion of patients with both chiasmatic and nerve involvement (44\% vs. $33 \%$ ); however, the difference between the groups was not statistically significant.

\subsection{Evolution of the two groups (Table 3, Fig. 1)}

One symptomatic OPG patient did not return for ophthalmologic or MRI assessment and was lost to follow-up. Longitudinal data averaged 7.8 years for asymptomatic patients (range 0.8-11.8 years) and 7.7 years for symptomatic patients (range 5.3-10 years). Table 3 and Fig. 1 summarize the evolution in both groups. Progression was observed in 16 cases. Radiological progression without clinical progression was observed in only one symptomatic patient. Clinical progression with or without radiographic progression was observed

Table 1 - General characteristics of the population.

\begin{tabular}{llcc} 
& Total population & \multicolumn{2}{c}{ Children with OPG } \\
\cline { 3 - 4 } & & Asymptomatic & Symptomatic \\
\hline Number & 306 & 36 & 9 \\
Age (mean) for NF1 diagnostic & $3 y$ & $3.3 y$ & $3.7 y$ \\
Sex ratio (M/F) & $158 / 148$ & $17 / 19$ & $3.4 \mathrm{y}$ \\
Age (mean) at screening & $3.4 \mathrm{y}$ & $3.4 \mathrm{y}$ & $23 / 22$ \\
Family history of NF1 n (\%) & $128(41.8 \%)$ & $14(38.8 \%)$ & $3.5 \mathrm{y}$ \\
\hline
\end{tabular}


Table 3 - Evolution for asymptomatic and symptomatic groups.

Asymptomatic Symptomatic P value

$$
(\mathrm{n}=36)
$$

$$
(\mathrm{n}=8)
$$

\section{Follow-up duration (y)}

Evolution n (\%)

No progression

Radiologic tumor

progression only

Clinical tumor progression

(with or without

radiologic

progression)

Visual function at the

end of follow-up $n$ (\%)

Normal

Abnormal (Mild)

Abnormal (Severe)

7,8

7,7

$0.750^{\mathrm{b}}$

$0.119^{a}$

$25(69.4 \%) \quad 3(33.3 \%)$

$0 \quad 1(11.1 \%)$

$11(30.5 \%) \quad 4(44.4 \%)$

\section{$\mathrm{y}=$ years.}

a Test Khi-2 of Pearson

b Non parametric test. temporal and brainstem involvement, with hydrocephaly. Temodal was initiated as palliative therapy and a ventriculoperitoneal shunt was placed. This patient was tetraparetic and blind. At the end of follow-up, 31 patients (70\%) had normal visual function, including 29 in the asymptomatic group and two in the symptomatic group. Thirteen patients (30\%) had mild to severe visual impairment, including seven in the asymptomatic group and six in the symptomatic group. The difference between the two groups was statistically significant (Table 3). All the patients treated with chemotherapy had severe visual outcome. No difference was observed in these patients for visual function at the beginning and at the end of treatment. One patient improved the Visual Evoked Potentials without improvement of visual functions. Details for the 13 patients with abnormal visual function at the end of follow-up are described in Table 4.

\section{Discussion}

This study confirmed the existing epidemiologic data on OPG in NF1 in the French population, which have already been reported in American populations ${ }^{7-9}$ : OPG concerned about $15 \%$ of NF1 children screened with MRI, a small proportion were symptomatic (around 3\% of NF1 children at the time of diagnosis and $5 \%$ during follow-up), and the preponderance in females reported by others authors ${ }^{5,9,10}$ was not observed in our study, which revealed a sex ratio close to $1: 1$. The average age at OPG diagnosis was nearly 3 years, as reported in previous studies with systematic MRI screening, ${ }^{4,11}$ which two years earlier than the age at diagnosis reported in large retrospective OPG studies of NF1 populations without systematic MRI screening. ${ }^{12}$ Thus, systematic MRI screening probably provides earlier OPG diagnosis.

Ophthalmologic signs were more frequent at the time of OPG diagnosis. The three most frequent ophthalmologic symptoms were decreased visual acuity (3), evidence of optic atrophy or swelling on funduscopy examination (2), or proptosis (1). One patient underwent imaging to investigate macrocephaly and one other had growth rate acceleration. King et al. $(2003)^{13}$ reported precocious puberty in $20 \%$ of patients and poor weight gain in $4 \%$ of children with NF1 and OPG. In this study, four patients (9\%) with chiasmatic involvement experienced precocious puberty during the course of OPG. When one of these symptoms was present, MRI revealed OPG in more than $50 \%$ of cases. In our study, of the six patients treated with chemotherapy, five had chiasmatic involvement, which confirms that patients with chiasmal involvement seem to be more susceptible to visual morbidity. ${ }^{9,11,14}$

Negative MRI does not rule out later emergence of OPG, as reported by other authors ${ }^{15,16}$ : two children presented symptomatic OPG after a first normal MRI. Moreover, newly diagnosed OPG may raise parental anxiety while most of the patients with incidentally discovered OPG will not experience any progression. These two points constitute arguments against systematic screening.

OPG progression after diagnosis was observed in $34 \%$ and more frequently in the symptomatic group; this is consistent with data published by other authors. ${ }^{17}$ Nevertheless, OPG progression in the asymptomatic group (30\%) was more

Fig. 1 - Flow chart of the study population. 


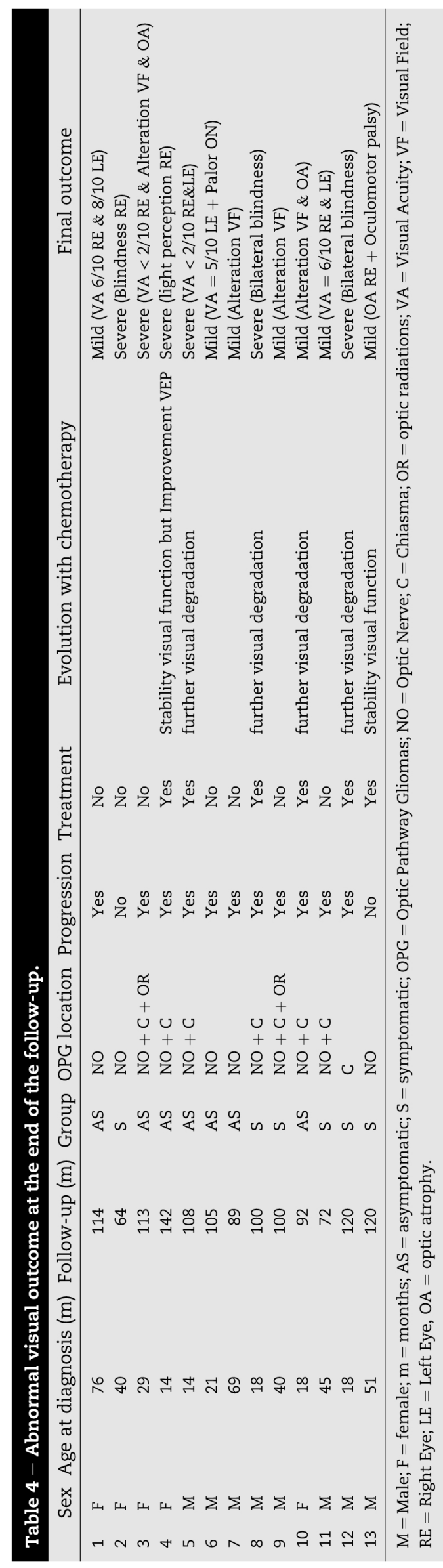

frequent than reported previously. ${ }^{3}$ However, clinical or radiological changes and real tumor progression were not correlated. Serial changes in visual function do not reliably detect tumor progression and conversely, radiological evidence of tumor progression does not reliably correlate with changes of visual function. ${ }^{18}$

At the end of follow-up, visual impairment concerned $30 \%$ of the children, which corresponds to other reports $(22 \%-31 \%)$ on more recent series. ${ }^{4,12,13}$ The percentage of children with visual impairment (mild or severe) at follow-up was significantly higher in the symptomatic group (6/8) compared to the asymptomatic group (7/36). Thiagalingam et al. ${ }^{12}$ reported $16.7 \%$ of patients having moderate to severe bilateral impairment. In the Blazo and Thiagalingam studies, patients systematically screened at OPG diagnosis did not have severe visual impairment at follow-up. Fisher et al. (2012) ${ }^{17}$ reported poorer prognosis when OPG involved posterior optic tracts: our study did not reveal differences in tumor location between the groups.

The range of visual acuity tests used on our patients at the different centers was a weak point in our study and highlights the difficulty of assessing visual function accurately in young children. The Response Evaluation in Neurofibromatosis and Schwannomatosis Visual Outcomes Committee recently defined visual function assessments using consistent quantitative testing methods as the best functional outcome measures for NF1-OPG clinical trials. Teller acuity cards are recommended for use as a primary visual acuity endpoint and HOTV as a secondary endpoint once subjects are old enough to complete it. They also propose evaluating visual quality of life using the Children's Visual Function Questionnaire as a secondary endpoint. ${ }^{19}$

Most patient conditions were managed conservatively $(87 \%)$. Six children $(13 \%)$ were treated with chemotherapy because of worsening visual function. All of these children had severe or mild visual impairment at the end of follow-up. In four children, visual function worsened despite chemotherapy and none had improved function. As mentioned by some authors, ${ }^{18}$ it is still unknown whether this worsening during chemotherapy reflects progression of the illness or damage occurring prior to treatment. Nevertheless, limited data exist regarding the clinical outcome of children with OPG after chemotherapy. ${ }^{20}$ Moreno et al. reviewed the literature systematically from 1990 to 2008 and reported the visual outcome in children with OPG who received chemotherapy. Of 85 potentially relevant publications, only eight were included. Dalla Via et al. (2007) ${ }^{21}$ described unsatisfactory visual outcome in children with NF1 and OPG treated by chemotherapy. Fisher et al. ${ }^{17}$ reported a retrospective multicenter study including 115 patients. Eighty-eight subjects and 168 eyes were evaluated for visual acuity outcome. After chemotherapy, $32 \%$ of children improved, $40 \%$ remained stable, and $28 \%$ worsened. The lack of correlation between visual and radiographic outcomes in this study argues against the use of MRI response as the gold standard of treatment for this tumor. Kalin-Hajdu et al. ${ }^{22}$ recently reported decreased visual acuity in seven NF1-OPG patients directly after chemotherapy and at long-term follow-up. Whether chemotherapy, when initiated due to a slight decline in visual function, is superior to conservative management needs to be demonstrated in NF1 children through a prospective, multicenter randomized study. 
In conclusion, our multicenter study does not support a clear benefit of systematic MRI screening in NF1 children under six years of age. Firstly, systematic neuroimaging in our study did not influence the therapeutic management of OPG patients since decreased visual acuity was the primary reason for initiating treatment. Secondly, while the OPG diagnosis was made early, treatment by chemotherapy did not improved the final visual outcome; this conclusion is in accordance with several studies questioning the efficacy of chemotherapy in children with NF1 and OPG. Thirdly, the majority of NF1 children who developed OPG did not require treatment and only children who had progressive visual deterioration needed treatment. To date, the best method of treatment has not been determined and probably new therapies targeting mTOR activity should be developed for treatment of tumors in NF1 patients. Finally, our study suggests that brain MRI should be dictated by the results of systematic annual, clinical and ophthalmological examinations. ${ }^{23}$ In this case, abnormal eye examinations or precocious puberty should lead to an investigation of OPG by MRI. ${ }^{24}$ We must remain cautious, of course: the examination should be complete and beyond doubt. In light of these results, the French recommendations on the follow-up of children with NF1 should be reviewed.

\section{Conflict of interest}

The authors declare no conflict of interest.

\section{Acknowledgments}

We thank the Association Neurofibromatoses et Recklinghausen for their support in this study.

We express our gratitude to the NF-France Network for its precious collaboration:

Pierre Wolkenstein, Laurence Allanore, Jean Christophe Moreno, Salah Ferkal, Diana Rodriguez Smaïl Hadj-Rabia, Stanislas Lyonnet, Pierre Vabres, Hélène Dollfus, Jean-François Cuny, Dominique Gaillard, Philippe Bernard, Michelle Senez, Jean-François Stalder, Jean-Sébastien Guillamo, Anne Dompmartin, Henri Adamski, Xavier Balguerie, Bernard Guillot, Juliette Mazereeuw Hautier, Frédérique Audic, Philippe Berbis, Jean-Philippe Lacour, Patrick Combemale, Stéphane Pinson, Michel d'Incan, Christine Francannet, Gérard Lorette, Marie-Noëlle loiseau, Jean-Marie Bonnetblanc, Ali Dadban, Jean Claude Léonard, Emmanuel Delaporte, Christian Derancourt.

\section{R E F E R E N C ES}

1. Viskochil D. Genetics of neurofibromatosis 1 and the NF1 gene. J Child Neurol 2002;17:562-70.

2. Neurofibromatosis. conference statement. National institutes of health consensus development conference. Arch Neurol $1988 ; 45: 575-8$.
3. Listernick R, Charrow J, Greenwald M, Mets M. Natural history of optic pathway tumors in children with neurofibromatosis type 1: a longitudinal study. J Pediatr 1994;125:63-6.

4. Blazo MA, Lewis RA, Chintagumpala MM, Frazier M, McCluggage C, Plon SE. Outcomes of systematic screening for optic pathway tumors in children with neurofibromatosis type 1. Am J Med Genet 2004;127:224-9. 1.

5. Listernick R, Louis DN, Packer RJ, Gutmann DH. Optic pathway gliomas in children with neurofibromatosis 1 : consensus statement from the NF1 optic pathway glioma task Force. Ann Neurol 1997;41:143-9.

6. Pinson S, Créange A, Barbarot S, Stalder JF, Chaix Y, Rodriguez D, Sanson M, Bernheim A, d'Incan M, Doz F, Stoll C, Combemale P, Kalifa C, Zeller J, Teillac-Hamel D, Lyonnet S, Zerah M, Lacour JP, Guillot B, Wolkenstein P.

Neurofibromatosis 1: recommendations for management. Arch Pediatr 2002;9:49-60.

7. Lewis RA, Gerson LP, Axelson KA, Riccardi VM, Whitford RP. Von Recklinghausen neurofibromatosis. II. Incidence of optic gliomata. Ophthalmology 1984;91:929-35.

8. Listernick R, Charrow J, Greenwald MJ, Esterly NB. Optic gliomas in children with neurofibromatosis type 1.J Pediatr 1989;114:788-92.

9. Listernick R, Darling C, Greenwald M, Strauss L, Charrow J. Optic pathway tumors in children: the effect of neurofibromatosis type 1 on clinical manifestations and natural history. J Pediatr 1995;127:718-22.

10. Diggs-Andrews KA, Brown JA, Gianino SM, Rubin JB, Wozniak DF, Gutmann DH. Sex is a major determinant of neuronal dysfunction in neurofibromatosis type 1. Ann Neurol 2014;75:309-16.

11. Balcer LJ, Liu GT, Heller G, Bilaniuk L, Volpe NJ, Galetta SL, Molloy PT, Phillips PC, Janss AJ, Vaughn S, Maguire MG. Visual loss in children with neurofibromatosis type 1 and optic pathway gliomas: relation to tumor location by magnetic resonance imaging. Am J Ophthalmol 2001;131:442-5.

12. Thiagalingam S, Flaherty M, Billson F, North K. Neurofibromatosis type 1 and optic pathway gliomas: followup of 54 patients. Ophthalmology 2004;111:568-77.

13. King A, Listernick R, Charrow J, Piersall L, Gutmann DH. Optic pathway gliomas in neurofibromatosis type 1: the effect of presenting symptoms on outcome. Am J Med Genet A 2003;122:95-9.

14. Deliganis AV, Geyer JR, Berger MS. Prognostic significance of type 1 neurofibromatosis (von Recklinghausen Disease) in childhood optic glioma. Neurosurgery 1996;38:1114-8.

15. Listernick Charrow J, Greenwald M. Emergence of optic pathway gliomas in children with neurofibromatosis type 1 after normal neuroimaging results. J Pediatr 1992;121:584-7.

16. Massry GC, Morgan CF, Chung SM. Evidence of optic pathway gliomas after previously negative neuroimaging. Ophthalmology 1997;104:930-5.

17. Fisher MJ, Loguidice M, Gutmann DH, Listernick R, Ferner RE, Ullrich NJ, Packer RJ, Tabori U, Hoffman RO, ArdernHolmes SL, Hummel TR, Hargrave DR, Bouffet E, Charrow J, Bilaniuk LT, Balcer LJ, Liu GT. Visual outcomes in children with neurofibromatosis type 1-associated optic pathway glioma following chemotherapy: a multicenter retrospective analysis. Neuro Oncol 2012;14:790-7.

18. Kelly JP, Weiss AH. Detection of tumor progression in optic pathway glioma with and without neurofibromatosis type 1 . Neuro Oncol 2013;15:1560-7.

19. Fisher MJ, Avery RA, Allen JC, Arden-Holmes SL, Bilaniuk LT, Ferner RE, Gutmann DH, Listernick R, Martin S, Ulrrich NJ, Liu GT. Functional outcome measures for NF1-associated optic pathway glioma clinical trials. Neurology 2013;81(Suppl 1):S15-24. 
20. Moreno L, Bautista F, Ashley S, Duncan C, Zacharoulis S. Does chemotherapy affect the visual outcome in children with optic pathway glioma? A systematic review of the evidence. Eur J Cancer 2010;46:2253-9.

21. Dalla Via P, Opocher E, Pinello ML, Calderone M, Viscardi E, Clementi M, Battistella PA, Laverda AM, Da Dalt L, Perilongo G. Visual outcome of a cohort of children with neurofibromatosis type 1 and optic pathway glioma followed by a pediatric neuro-oncology program. Neuro Oncol 2007;9:430-7.
22. Kalin-Hajdu E, Décarie JC, Marzouki M, Garret AS, Ospina LH Visual acuity of children treated with chemotherapy for optic pathway gliomas. Pediatr Blood Cancer 2014;61:223-7.

23. Hirbe AC, Gutmann DH. Neurofibromatosis type 1: a multidisciplinary approach to care. Lancet Neurol 2014;13:834-43.

24. Listernick R, Ferner RE, Liu GT, Gutmann DH. Optic pathway gliomas in neurofibromatosis-1: controversies and recommandations. Ann Neurol 2007;61:189-98. 